\title{
Hormone and androgen receptor genes combined affect sperm motility
}

Published online: 01 October 2012

(C) Springer Healthcare 2012

medwireNews: The combination of alleles for genes encoding the follicle-stimulating hormone receptor (FSHR) and androgen receptor (AR) has a significant impact on sperm motility, emphasising the importance of the genes in sperm quality, say Greek scientists.

The team, led by Ioannis Georgiou, from Ioannina University, comments: "The most interesting result of the current study is the synergistic effect of FSHR and AR genetic variants on sperm motility... Men with long AR alleles presented higher sperm motility compared with short AR allele carriers, with a gradual reduction from Thr307Thr/Asn680Asn to Thr307Ala/Asn680Ser and Ala307Ala/ Ser680Ser diplotype carriers in each group.”

Studying 200 oligozoospermic and 250 normozoospermic men referred to an in vitro fertilization unit, the team initially found that there were three diplotypes of the FSHR polymorphisms: Thr307Thr/Asn680Asn; Thr307Ala/ Asn680Ser; and Ala307Ala/Ser680Ser. There were no significant differences in proportions of the diplotypes between the two patient groups.

Turning their attention to the $\mathrm{AR}\left(\mathrm{CAG}_{\mathrm{n}}\right)$ polymorphism, the researchers demonstrated that there were 18 alleles with $12-32$ repeats, which could be categorized into short and long AR alleles. Again, the repeat length of the AR alleles did not differ significantly between the two groups.

FSHR diplotypes were not associated with serum hormone levels, sperm concentration, and motility, whereas AR $\left(\mathrm{CAG}_{\mathrm{n}}\right)$ genotypes correlated with sperm motility. Specifically, longer AR alleles were associated with higher sperm motility than shorter alleles in the normospermic group, at $57.9 \%$ versus $45.9 \%$.

Combined FSHR-AR genotypes were significantly associated with sperm motility in the normospermic group, at $62.2 \%, 48.9 \%, 47.9 \%$, and $41.8 \%$ for long AR alleleThr307Thr/Asn680Asn carriers, long AR allele-Ala307Ala/Ser680Ser carriers, short AR alleleThr307Thr/Asn680Asn carriers, and short AR allele- Ala307Ala/Ser68oSer carriers, respectively. Similar findings were observed in oligozoospermic men, at 58.1\%, 40.8\%, 41.6\%, and $25.7 \%$, respectively.

By Liam Davenport, medwireNews Reporter

\section{Reference}

Andrologia 2012; Advance online publication 